\title{
Factores asociados a la fragilidad en el paciente en tratamiento renal sustitutivo con diálisis. Una revisión sistemática
}

\author{
Olga Cobacho-Salmoral ${ }^{1}$, Lucía Parra-Martos ${ }^{1}$, Marta Laguna-Castro ${ }^{1}$, Rodolfo Crespo-Montero $0^{1,2,3}$ \\ ${ }^{1}$ Departamento de Enfermería. Facultad de Medicina y Enfermería. Universidad de Córdoba. España \\ ${ }^{2}$ Servicio de Nefrología. Hospital Universitario Reina Sofía de Córdoba. España \\ ${ }^{3}$ Instituto Maimónides de Investigación Biomédica de Córdoba. España
}

\begin{abstract}
Como citar este artículo: Cobacho-Salmoral 0, Parra-Martos L, Laguna-Castro M, Crespo-Montero R. Factres asociados a la fragilidad en el paciente en tratamiento renal sustitutivo con diálisis. Una revisión sistemática. Enferm Nefrol. 2021 Jul-Sep;24(3):233-48
\end{abstract}

\section{Resumen}

Introducción: La fragilidad es un síndrome multidimensional caracterizado por una disminución de reservas fisiológicas, de masa corporal magra, debilidad y disminución de resistencia al ejercicio físico. Sitúa a la persona en una situación de mayor vulnerabilidad ante factores externos, además existe una estrecha relación entre la fragilidad y las enfermedades crónicas, como es el caso de la enfermedad renal crónica.

Objetivo: El objetivo principal de esta revisión fue sintetizar y conocer la evidencia científica sobre los factores asociados a la fragilidad de los pacientes con enfermedad renal crónica en tratamiento renal sustitutivo en diálisis.

Material y Método: Se llevó a cabo una revisión sistemática a través de las bases de datos de Pubmed y Proquest. Se incluyeron artículos originales en inglés y español publicados entre 2015 y 2020, y se analizaron los artículos que trataban sobre fragilidad en el paciente con enfermedad renal en tratamiento sustitutivo. Se excluyeron aquellos artículos que no presentaron resultados.

\section{Correspondencia:}

Olga Cobacho Salmoral

Email: olgacobach097@gmail.com
Resultados: Se incluyeron 26 artículos de diseño observacional. Los hallazgos se enfocan a la prevalencia, mortalidad, tasa de hospitalización, discapacidad, deterioro cognitivo, síntomas depresivos, obesidad, comorbilidades, caídas o fracturas y actividad y rendimiento físico.

Conclusiones: Se ha encontrado una elevada prevalencia de fragilidad en el enfermo renal crónico en tratamiento renal sustitutivo con diálisis, sin diferencias entre diálisis peritoneal y hemodiálisis. La fragilidad en estos pacientes está asociada a mayor mortalidad, tasa de hospitalización, discapacidad, deterioro cognitivo, síntomas depresivos y comorbilidades. La actividad y rendimiento físico, parecen ser factores que disminuyen la fragilidad.

PALABRAS CLAVE: fragilidad; enfermedad renal crónica; tratamiento renal sustitutivo; diálisis peritoneal; hemodiálisis.

Factors associated with frailty in patients on renal replacement therapy with dialysis. A systematic review

\section{Abstract}

Introduction: Frailty is a multidimensional syndrome characterized by a decrease in physiological reserves, lean body mass, weakness and decreased resistance 
to physical exercise. It places the person in a situation of greater vulnerability to external factors, in addition there is a close relationship between frailty and chronic diseases, such as renal insufficiency chronic.

Objective: The main objective of this review was to synthesize and know the scientific evidence on the factors associated with the frailty of patients with renal insufficiency chronic in renal replacement therapy with dialysis.

Material and Method: A systematic review was carried out using the PubMed and ProQuest databases. Original articles in English and Spanish published between 2015 and 2020 were included, and articles dealing with frailty in patients with renal disease on renal replacement therapy were analysed. Articles that did not present results were excluded.

Results: 27 observational design articles were included. The findings focus on prevalence, mortality, hospitalization rate, disability, cognitive decline, depressive symptoms, obesity, comorbidities, falls or fractures, and physical activity.

Conclusions: A high prevalence of frailty has been found in the chronic renal patient in replacement therapy with dialysis, without differences between peritoneal dialysis and hemodialysis. Frailty in these patients is associated with higher mortality, hospitalization rate, disability, cognitive decline, depressive symptoms and comorbidities. Physical activity and performance appear to be factors that decrease frailty.

KEYWORDS: frailty; chronic kidney disease; renal replacement therapy; peritoneal dialysis; hemodialysis.

\section{Introducción}

La fragilidad se define como un síndrome multidimensional caracterizado por una pérdida de reservas fisiológicas, disminución de masa corporal magra, debilidad y disminución de la resistencia al ejercicio físico ${ }^{1,2}$, lo que sitúa a la persona en una situación de mayor vulnerabilidad ante factores externos (estresantes) y por lo tanto mayor posibilidad de eventos adversos de salud como la hospitalización, institucionalización, muerte y caídas ${ }^{3,4}$.

El concepto de fragilidad es diferente al de envejecimiento, la discapacidad y la comorbilidad, aunque está claramente relacionada con estos factores. Además, aun- que la prevalencia de la fragilidad aumenta con la edad, ocurre independientemente de la edad cronológica ${ }^{5}$.

Según diferentes autores, existe una estrecha relación entre la fragilidad y las enfermedades crónicas que acompañan a la edad avanzada, con elementos contribuyentes entre ambas. Las enfermedades crónicas pueden contribuir como factor causal o precipitante del síndrome frágil, a la vez que éste puede condicionar mayor vulnerabilidad y complicaciones en los adultos mayores que padecen enfermedades crónicas de base, como ocurre en la enfermedad renal ${ }^{6}$.

Por tanto, en la enfermedad renal crónica (ERC) la prevalencia de fragilidad es alta, y aumenta con la edad y con el deterioro de la función renal ${ }^{7,8}$, presentando mayor prevalencia los pacientes que precisan una Terapia Renal Sustitutiva (TRS), sobre todo con diálisis. En estos pacientes, la prevalencia de fragilidad oscila entre el $26 \%$ y el $68 \%$, dependiendo de las características del grupo de estudio y de los instrumentos de detección de fragilidad que se utilizaron $3,9,10$.

Por ello, es muy importante como se identifica la fragilidad. En una revisión reciente, se ofrece una descripción general de las definiciones y la medición de la fragilidad en la investigación y la práctica clínica, y se concluye que las dos medidas más comunes son: el fenotipo de fragilidad de Fried y el Índice de fragilidad de Rockwood y Mitnitski ${ }^{5}$. Aunque la mayoría de los estudios actuales se basan en los criterios de Fried et al. o su adaptación; en 2001, su grupo propuso una definición de fenotipo de fragilidad, que se basa en las siguientes características: pérdida involuntaria de peso, agotamiento, lentitud en la movilidad, debilidad muscular y bajo nivel de actividad física ${ }^{11}$.

Teniendo en cuenta, que la ERC es más prevalente en personas mayores, y que esta población además tiene mayor porcentaje de comorbilidades, es lógico pensar que estos pacientes sean más frágiles; de hecho, una revisión sistemática encontró una gran diferencia entre la fragilidad en pacientes con ERC avanzada (15$21 \%$ ) y la población general $(3-6 \%)^{12}$. Se han sugerido varios mecanismos para explicar este vínculo, uno de ellos, denominado la "ineficiencia castigada", propone que tener deficiencias severas en un sistema, secundarias a una enfermedad, genera estrés e ineficiencias en otros sistemas, lo que resulta en desuso, deterioro físico y fragilidad ${ }^{13}$. En este sentido, Walker, Gill y Macdonald mostraron en una revisión que la ERC se asocia con un mayor riesgo de fragilidad y esta conduce a un mayor 
riesgo de mortalidad ${ }^{14}$. Por todo esto, y dado que la prevalencia de la ERC se ha asociado al aumento de edad de la población general, es lógico también pensar que esta población debe tener una alta prevalencia de fragilidad, motivo por el cual nos hemos planteado llevar a cabo esta revisión de la bibliografía más actualizada.

Por consiguiente, el objetivo principal de esta revisión fue sintetizar y conocer la evidencia científica sobre los factores asociados a la fragilidad en el paciente en TRS con diálisis, con los siguientes objetivos secundarios:

1. Determinar la prevalencia de la fragilidad en el paciente en TRS con diálisis.

2. Revisar los instrumentos más utilizados en los estudios sobre fragilidad en los pacientes en diálisis.

\section{Material y Método}

\section{Diseño}

Se ha llevado a cabo una revisión sistemática de estudios indexados en bases de datos de ciencias de la salud. Se ha realizado una síntesis temática categorial de los artículos seleccionados.

La revisión sistemática se ha desarrollado en base a la declaración PRISMA (Preferred Reporting Items for Systematic Reviews and Meta-Analyses) ${ }^{15}$ : (i) formulación la pregunta de investigación de acuerdo con la estructura PIC0: población, intervención, comparador y outcome (resultados); (ii) planteamiento de la estrategia de búsqueda para la identificación de estudios; (iii) extracción de datos; (iv) evaluación de la calidad de los estudios; y (v) análisis de los datos obtenidos.

\section{Métodos de búsqueda}

Las bases de datos empleadas fueron PubMed y Proquest. La estrategia de búsqueda se estableció con los siguientes términos MeSH: "frailty", "renal insufficiency", "renal insufficiency, chronic", "kidney diseases", "kidney treatment" y "renal replacement therapy". Los términos se combinaron con los operadores booleanos AND y OR, siendo la estrategia la siguiente: ( "renal insufficiency") OR ("renal insufficiency, chronic") OR ("kidney diseases") OR ("kidney treatment") OR ("renal replacement therapy")) AND ("frailty").

\section{Criterios de inclusión y exclusión}

Los criterios de inclusión y exclusión establecidos fueron: Criterios de inclusión:

- Artículos originales, que evaluaran fragilidad en pacientes con ERC en TRS con diálisis.
- Artículos en español y en inglés.

- Documentos publicados entre los años 2015 y 2020.

Criterios de exclusión:

- Artículos realizados en pacientes trasplantados renales.

- Artículos que no aportaran resultados.

\section{Resultados de búsqueda}

Una vez establecida la estrategia de búsqueda en las diferentes bases de datos seleccionadas, se procedió a la búsqueda de artículos desde el día 20 de octubre de 2020 al 25 de noviembre de 2020. De acuerdo a las recomendaciones de Moher et al. (2016) ${ }^{16}$, el proceso de búsqueda de estudios se plasmó en un diagrama de flujo PRISMA (figura 1).

Al incluir la estrategia de búsqueda en las bases de datos referidas se encontraron 217 artículos. Se eliminaron los manuscritos duplicados (12), quedando 205 para la lectura de título y resumen. Una vez revisado el título y resumen se eliminaron 133 manuscritos, quedando para lectura completa 72 artículos. Tras la lectura a texto completo, se excluyeron 46 artículos de los 72 . Finalmente fueron 26 los artículos seleccionados.

\section{Evaluación de la calidad de los artículos}

La calidad de los artículos seleccionados se determinó de acuerdo con la lista de verificación establecida por STROBE ${ }^{17}$ para los estudios observacionales. Además, se complementó con el índice de impacto de las revistas de las que fueron extraídos los artículos utilizados del Scimago Journal \& Country Rank (SJR) ${ }^{18}$, elaborado a partir de la base de datos de Scopus, para tener una aproximación de la calidad de la revista en la que estaban publicados los artículos según este índice.

\section{Extracción de datos}

Se extrajeron las siguientes variables de los artículos seleccionados: autor, año, país, tipo de estudio, muestra, procedimiento dialítico, instrumento de medida de fragilidad, resultados principales y calidad de la evidencia.

\section{Síntesis de resultados}

Se seleccionaron 26 artículos para la presente revisión sistemática, según los criterios de inclusión y exclusión establecidos. Se realizó un enfoque descriptivo-categorial debido a la existencia de heterogeneidad de resultados y variables analizados en los artículos estudiados. 


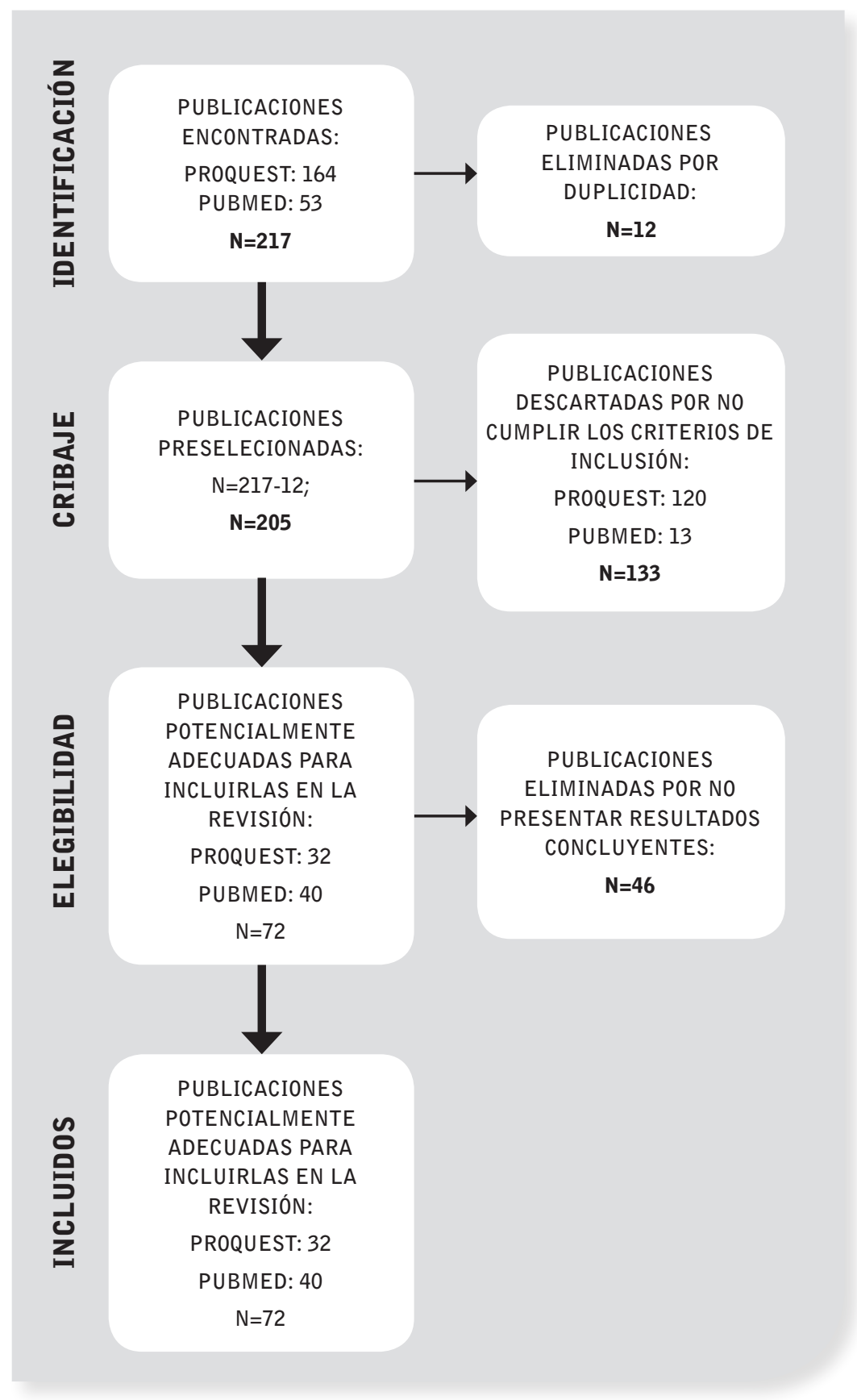

Figura 1. Diagrama de flujo de la estrategia de búsqueda.

\section{Descripción de los resultados}

Los resultados de los artículos se han clasificado según el tipo de tratamiento que reciben los pacientes: estudios en los que se analizan los datos de pacientes en diálisis, tanto en Hemodiálisis (HD) como en Diálisis Peritoneal (DP), pacientes que estaban solo en tratamiento con $\mathrm{HD}$, pacientes que estaban en tratamiento en DP, y pacientes en diálisis incluidos en lista de espera para trasplante renal (TR), independientemente del procedimiento dialítico en el que estuvieran.

\section{Prevalencia}

La prevalencia media de fragilidad en los estudios revisados de pacientes en diálisis en los cuales la población estuvo en alguna de las 2 modalidades (HD o DP), sin diferenciar el procedimiento fue del $45,4 \% 20,21,38,39$.

La fragilidad de los pacientes en HD, recogida en 10 artículos, se presentó en el $38,04 \%{ }^{24-27,30,32-36}$. En un artículo se midió fragilidad de dos formas, según el cuestionario de Fried, la prevalencia era del $31 \%$, y según el cuestionario de autoinforme de función física (contestado por el propio paciente) era del $52 \%{ }^{27}$. En un estudio sobre la asociación de fragilidad autoinformada con caídas y fracturas también se midió con este cuestionario autoinformado y la prevalencia fue del $77 \%{ }^{28}$. Solo en un estudio trasversal se analizó la fragilidad percibida y medida entre adultos sometidos a HD, entre los pacientes frágiles solo el $42 \%$ fueron percibidos correctamente por su nefrólogo y $39,2 \%$ por su enfermera. Solo $4,9 \%$ se percibían frágiles a sí mismos, por lo que la fragilidad percibida no es un indicador adecuado de medida de fragilidad ${ }^{23}$.

\section{Resultados}

\section{Características de los estudios}

Los 26 artículos incluidos en esta revisión son de diseño observacional, de los cuales 17 son estudios longitudinales de cohorte prospectivo, 5 son estudios longitudinales de cohorte retrospectivo y 4 son estudios de corte transversal. Los artículos seleccionados y sus características se pueden observar en la tabla 1.
En los 4 estudios en pacientes que se dializaban con DP, la fragilidad se presentó en el $42,15 \%$ los pacientes ${ }^{22,29,30,37}$.

En los estudios de los pacientes en diálisis que se encuentran en lista de espera para ser trasplantados de riñón, la fragilidad se midió en algunos casos meses antes del trasplante y en otros en el momento de ingreso para trasplantarse, encontrándose un $22,78 \%$ de pacientes frágiles en 5 estudios ${ }^{40-44}$. 
Tabla 1. Características de los artículos seleccionados para la revisión.

\begin{tabular}{|c|c|c|c|c|c|c|}
\hline $\begin{array}{l}\text { Auto; Año; } \\
\text { País }\end{array}$ & Tipo de estudio & Muestra & $\begin{array}{l}\text { Tipo de } \\
\text { Tratamiento }\end{array}$ & $\begin{array}{l}\text { Instrumento } \\
\text { de medida }\end{array}$ & Resultados & $\begin{array}{l}\text { Criterio de } \\
\text { calidad }\end{array}$ \\
\hline $\begin{array}{l}\text { Kang et al. }{ }^{19} \text {; } \\
\text { 2017; Corea. }\end{array}$ & $\begin{array}{l}\text { Estudio longitudinal } \\
\text { de cohorte } \\
\text { retrospectivo }\end{array}$ & 1.611 & $\begin{array}{l}\text { HD (364) y DP } \\
\text { (1247) }\end{array}$ & KDQ0L-SF-36 & $\begin{array}{l}\text { La proporción de pacientes con } \\
\text { fragilidad y la presencia de cada } \\
\text { componente (lentitud, poca resistencia } \\
\text { y pérdida de peso no intencionada) } \\
\text { disminuyó a medida que aumentó la } \\
\text { actividad física. }\end{array}$ & $\begin{array}{l}\text { STROBE: } \\
21 / 22 \\
\text { SJR: Q2 }\end{array}$ \\
\hline $\begin{array}{l}\text { Lee et al. }{ }^{20} \text {; } \\
\text { 2016; Corea } \\
\text { del Sur. }\end{array}$ & $\begin{array}{l}\text { Estudio longitudinal } \\
\text { de cohorte } \\
\text { retrospectivo }\end{array}$ & 1.658 & $\begin{array}{l}\text { HD (1255) y DP } \\
(403)\end{array}$ & KDQ0L-SF-36 & $\begin{array}{l}\text { El fenotipo de fragilidad fue común } \\
\text { incluso en pacientes prevalentes con } \\
\text { enfermedad renal en etapa terminal } \\
\text { en diálisis, y fue significativamente } \\
\text { asociado con mayores tasas de } \\
\text { hospitalización y mortalidad. }\end{array}$ & $\begin{array}{l}\text { STROBE: } \\
\text { 21/22 } \\
\text { SJR: Q1 }\end{array}$ \\
\hline $\begin{array}{l}\text { Van Loon et } \\
\text { al. }^{21} ; 2019 ; \\
\text { Países Bajos. }\end{array}$ & $\begin{array}{l}\text { Estudio longitudinal } \\
\text { de cohorte } \\
\text { retrospectivo }\end{array}$ & 281 & $\begin{array}{l}\text { HD (148) } \\
\text { DP (44) } \\
\text { Terapia de manejo } \\
\text { conservador (89) }\end{array}$ & $\begin{array}{l}\text { Valoración } \\
\text { geriátrica }\end{array}$ & $\begin{array}{l}\text { El } 88 \% \text { de los pacientes en tratamiento } \\
\text { conservador presentaron fragilidad } \\
\text { frente al } 78 \% \text { de los pacientes } \\
\text { en diálisis }(p=0,06) \text {. La tasa de } \\
\text { hospitalización fue mayor en los } \\
\text { pacientes que iniciaron diálisis. }\end{array}$ & $\begin{array}{l}\text { STROBE: } \\
22 / 22 \\
\text { SJR: Q2 }\end{array}$ \\
\hline $\begin{array}{l}\text { Yi et al.22; } \\
\text { 2018; China }\end{array}$ & $\begin{array}{l}\text { Estudio longitudinal } \\
\text { de cohorte } \\
\text { retrospectivo }\end{array}$ & 784 & DPCA & $\begin{array}{l}\text { Escala de } \\
\text { fragilidad } \\
\text { clínica (EFC) }\end{array}$ & $\begin{array}{l}\text { La fragilidad coexistente y el deterioro } \\
\text { cognitivo se asoció con una disminución } \\
\text { de la tasa de supervivencia y aumento } \\
\text { de la tasa de peritonitis. }\end{array}$ & $\begin{array}{l}\text { STROBE: } \\
20 / 22 \\
\text { SJR: Q1 }\end{array}$ \\
\hline $\begin{array}{l}\text { Salter et } \\
\text { al. }{ }^{23} ; 2015 ; \\
\text { Maryland, } \\
\text { EE.UU. }\end{array}$ & Estudio transversal & 146 & HD & $\begin{array}{l}\text { Opinión } \\
\text { profesional } \\
\text { y paciente } \\
\text { (fragilidad } \\
\text { percibida) } \\
\text { Criterios de } \\
\text { Fried }\end{array}$ & $\begin{array}{l}\text { La fragilidad percibida por el nefrólogo, } \\
\text { enfermera y el propio paciente es un } \\
\text { indicador inadecuado de la fragilidad } \\
\text { medida entre los pacientes sometidos } \\
\text { a hemodiálisis. Entre los participantes } \\
\text { no frágiles, el } 34,4 \%, 30,0 \% \text { y } 31,6 \% \\
\text { fueron percibidos como frágiles por un } \\
\text { nefrólogo, enfermera o ellos mismos. }\end{array}$ & $\begin{array}{l}\text { STROBE: } \\
21 / 22 \\
\text { SJR: Q1 }\end{array}$ \\
\hline $\begin{array}{l}\text { Chiang } \\
\text { et al.24; } \\
\text { 2018; San } \\
\text { Francisco, } \\
\text { EE.UU. }\end{array}$ & $\begin{array}{l}\text { Estudio longitudinal } \\
\text { de cohorte prospectivo }\end{array}$ & 440 & HD & $\begin{array}{l}\text { Criterios de } \\
\text { Fried }\end{array}$ & $\begin{array}{l}\text { Una concentración } 50 \% \text { menor de } \\
\text { testosterona libre se asoció con } 1,40 \\
\text { veces más probabilidades de ser frágil } \\
\text { y } 1,40 \text { veces de probabilidades más } \\
\text { altas de convertirse en frágil durante } \\
12 \text { meses. } \\
\text { Se comprobó mediante dos } \\
\text { componentes de la fragilidad: fuerza de } \\
\text { agarre y velocidad de la marcha. }\end{array}$ & $\begin{array}{l}\text { STROBE: } \\
\text { 22/22 } \\
\text { SJR: Q1 }\end{array}$ \\
\hline $\begin{array}{l}\text { Yadla et al. }{ }^{25} \text {; } \\
\text { 2017; India }\end{array}$ & $\begin{array}{l}\text { Estudio longitudinal } \\
\text { de cohorte prospectivo }\end{array}$ & 205 & HD & $\begin{array}{l}\text { Criterios de } \\
\text { Fried }\end{array}$ & $\begin{array}{l}\text { La fragilidad estuvo presente en el } \\
82 \% \text { de la población del estudio. } \\
\text { La fragilidad es un factor de } \\
\text { riesgo importante para las caídas y } \\
\text { hospitalizaciones. }\end{array}$ & $\begin{array}{l}\text { STROBE: } \\
20 / 22 \\
\text { SJR: Q3 }\end{array}$ \\
\hline $\begin{array}{l}\text { Yoneki et } \\
\text { al. }{ }^{26} ; 2018 ; \\
\text { Japón. }\end{array}$ & Estudio transversal & 214 & HD & $\begin{array}{l}\text { Criterios de } \\
\text { Fried }\end{array}$ & $\begin{array}{l}\text { La fragilidad fue asociada con los } \\
\text { parámetros del calcáneo: velocidad baja } \\
\text { del sonido, atenuación de ultrasonido } \\
\text { de banda ancha e índice de rigidez del } \\
\text { hueso. Los parámetros disminuyeron } \\
\text { con el aumento de los niveles de } \\
\text { fragilidad para ambos sexos. }\end{array}$ & $\begin{array}{l}\text { STROBE: } \\
20 / 22 \\
\text { SJR: Q2 }\end{array}$ \\
\hline
\end{tabular}




\begin{tabular}{|c|c|c|c|c|c|c|}
\hline $\begin{array}{l}\text { Auto; Año; } \\
\text { País }\end{array}$ & Tipo de estudio & Muestra & $\begin{array}{l}\text { Tipo de } \\
\text { Tratamiento }\end{array}$ & $\begin{array}{l}\text { Instrumento } \\
\text { de medida }\end{array}$ & Resultados & $\begin{array}{l}\text { Criterio de } \\
\text { calidad }\end{array}$ \\
\hline $\begin{array}{l}\text { Johansen et } \\
\text { al.27; 2016; } \\
\text { California, } \\
\text { EE.UU. }\end{array}$ & Estudio transversal & 762 & HD & $\begin{array}{l}\text { Criterios de } \\
\text { Fried } \\
\text { Autoinforme de } \\
\text { función física }\end{array}$ & $\begin{array}{l}\text { La fragilidad definida mediante } \\
\text { pruebas directas de rendimiento físico } \\
\text { y autoinforme de función física se } \\
\text { asoció con una mayor mortalidad entre } \\
\text { los pacientes que cumplían ambas } \\
\text { definiciones. No tenían riesgo de } \\
\text { mortalidad aquellos que solo cumplían } \\
\text { con la definición del autoinforme y } \\
\text { los que no eran frágiles por ninguna } \\
\text { definición. }\end{array}$ & $\begin{array}{l}\text { STROBE: } \\
21 / 22 \\
\text { SJR: Q1 }\end{array}$ \\
\hline $\begin{array}{l}\text { Delgado et } \\
\text { al.28; 2016; } \\
\text { California, } \\
\text { EE.UU. }\end{array}$ & $\begin{array}{l}\text { Estudio longitudinal } \\
\text { de cohorte prospectivo }\end{array}$ & 1053 & $H D$ & $\begin{array}{l}\text { Cuestionario } \\
\text { autoinformado, } \\
\text { adaptado por } \\
\text { criterios de } \\
\text { Fried }\end{array}$ & $\begin{array}{l}\text { La fragilidad autoinformada se asoció } \\
\text { con un mayor riesgo de caídas o } \\
\text { fracturas entre los pacientes nuevos en } \\
\text { diálisis. Tenían el doble de riesgo de } \\
\text { caídas o fracturas en comparación a los } \\
\text { que no. }\end{array}$ & $\begin{array}{l}\text { STROBE } \\
22 / 22 \\
\text { SJR: Q1 }\end{array}$ \\
\hline $\begin{array}{l}\text { Szeto et al. }{ }^{29} \text {; } \\
\text { 2018; China }\end{array}$ & $\begin{array}{l}\text { Estudio longitudinal } \\
\text { de cohorte prospectivo }\end{array}$ & 178 & DP & $\begin{array}{l}\text { Cuestionario } \\
\text { de fragilidad } \\
\text { validado por el } \\
\text { grupo }\end{array}$ & $\begin{array}{l}\text { El } 62,4 \% \text { de los pacientes eran } \\
\text { físicamente frágiles, entre ellos el } \\
43,2 \% \text { tenían síntomas depresivos. } \\
\text { Fragilidad física y síntomas depresivos } \\
\text { tienen efectos adversos aditivos sobre } \\
\text { el estado nutricional y sobre resultados } \\
\text { clínicos (menor supervivencia y mayor } \\
\text { tasa de hospitalización). }\end{array}$ & $\begin{array}{l}\text { STROBE: } \\
\text { 21/22 } \\
\text { SJR: Q2 }\end{array}$ \\
\hline $\begin{array}{l}\text { Kang et al. }{ }^{30} \text {; } \\
\text { 2017: Corea. }\end{array}$ & $\begin{array}{l}\text { Estudio longitudinal } \\
\text { de cohorte prospectivo }\end{array}$ & 1616 & $\begin{array}{l}\text { HD (1250) y DP } \\
(366)\end{array}$ & $\begin{array}{l}\text { CVRS: KDQOL- } \\
\text { SF } \\
\text { Fragilidad: PF } \\
\text { (funcionamien- } \\
\text { to físico) y VT } \\
\text { (vitalidad) de la } \\
\text { escala SF-36 }\end{array}$ & $\begin{array}{l}\text { No existen diferencias de prevalencia } \\
\text { de fragilidad entre ambos tratamientos. } \\
\text { La discapacidad fue más común en } \\
\text { los pacientes en DP }(29,8 \%) \text { que } \\
\text { en los pacientes en HD }(15,6 \%) \text {. } \\
\text { Se asoció fragilidad inversamente } \\
\text { con la supervivencia del paciente y } \\
\text { supervivencia libre de hospitalización en } \\
\text { los análisis multivariados en HD y DP. }\end{array}$ & $\begin{array}{l}\text { STROBE: } \\
20 / 22 \\
\text { SJR: Q1 }\end{array}$ \\
\hline $\begin{array}{l}\text { Johansen et } \\
\text { al.31; } 2017 ; \\
\text { California, } \\
\text { EE.UU. }\end{array}$ & $\begin{array}{l}\text { Estudio longitudinal } \\
\text { de cohorte prospectivo }\end{array}$ & 762 & $H D$ & $\begin{array}{l}\text { Criterios de } \\
\text { Fried }\end{array}$ & $\begin{array}{l}\text { Pacientes mayores, mujeres, hispanos } \\
\text { y diabéticos tenían más probabilidad } \\
\text { de ser frágiles, solo los marcadores de } \\
\text { inflamación y hospitalización fueron } \\
\text { independientemente asociados con el } \\
\text { empeoramiento de la fragilidad. }\end{array}$ & $\begin{array}{l}\text { STROBE: } \\
20 / 22 \\
\text { SJR: Q1 }\end{array}$ \\
\hline $\begin{array}{l}\text { 32. Johansen } \\
\text { et al. }{ }^{32} ; 2018 \text {; } \\
\text { California, } \\
\text { EE.UU. }\end{array}$ & $\begin{array}{l}\text { Estudio longitudinal } \\
\text { de cohorte } \\
\text { retrospectivo }\end{array}$ & 727 & HD & $\begin{array}{l}\text { Criterios de } \\
\text { Fried }\end{array}$ & $\begin{array}{l}\text { La actividad física y la velocidad de la } \\
\text { marcha disminuyeron, el agotamiento y } \\
\text { la fuerza de agarre no cambiaron, y las } \\
\text { probabilidades de alcanzar la pérdida } \\
\text { de peso declinó con el tiempo. Todos los } \\
\text { componentes de la fragilidad, excepto la } \\
\text { inactividad física, se asociaron de forma } \\
\text { independiente con la mortalidad cuando } \\
\text { los cinco componentes se incluyeron en } \\
\text { el mismo modelo. }\end{array}$ & $\begin{array}{l}\text { STROBE: } \\
21 / 22 \\
\text { SJR: Q1 }\end{array}$ \\
\hline $\begin{array}{l}\text { Gesualdo GD } \\
\text { et al. }{ }^{33} ; 2016 \text {; } \\
\text { Brasil. }\end{array}$ & $\begin{array}{l}\text { Estudio transversal } \\
\text { correlacional }\end{array}$ & 60 & HD & $\begin{array}{l}\text { Escala de } \\
\text { fragilidad de } \\
\text { Edmonton }\end{array}$ & $\begin{array}{l}\text { Se encontró que solo el ingreso mensual } \\
\text { per cápita se asoció significativamente } \\
\text { con fragilidad ( } 0 R=0,44 ; \text { IC: } 95 \% 0,1- \\
0,9 ; p=0,04 \text { ). Los ancianos con mayor } \\
\text { riesgo de la fragilidad eran aquellos con } \\
\text { menores ingresos. }\end{array}$ & $\begin{array}{l}\text { STROBE: } \\
20 / 22 \\
\text { SJR: Q2 }\end{array}$ \\
\hline
\end{tabular}




\begin{tabular}{|c|c|c|c|c|c|c|}
\hline $\begin{array}{l}\text { Auto; Año; } \\
\text { País }\end{array}$ & Tipo de estudio & Muestra & $\begin{array}{l}\text { Tipo de } \\
\text { Tratamiento }\end{array}$ & $\begin{array}{l}\text { Instrumento } \\
\text { de medida }\end{array}$ & Resultados & $\begin{array}{l}\text { Criterio de } \\
\text { calidad }\end{array}$ \\
\hline $\begin{array}{l}\text { McAdams- } \\
\text { DeMarco et } \\
\text { al. }{ }^{34} ; 2015 ; \\
\text { Canada. }\end{array}$ & $\begin{array}{l}\text { Estudio longitudinal } \\
\text { de cohorte prospectivo }\end{array}$ & 324 & HD & $\begin{array}{l}\text { Criterios de } \\
\text { Fried }\end{array}$ & $\begin{array}{l}\text { En pacientes adultos con HD incidente, } \\
\text { la fragilidad se asocia con una peor } \\
\text { función cognitiva, particularmente } \\
\text { función cognitiva global ( } 3 \mathrm{MS} \text { ). }\end{array}$ & $\begin{array}{l}\text { STROBE: } \\
20 / 22 \\
\text { SJR: Q1 }\end{array}$ \\
\hline $\begin{array}{l}\text { García Cantón } \\
\text { et al. } .^{35} ; 2019 ; \\
\text { España. }\end{array}$ & $\begin{array}{l}\text { Estudio longitudinal } \\
\text { de cohorte prospectivo }\end{array}$ & 277 & HD & $\begin{array}{l}\text { Escala de } \\
\text { fragilidad de } \\
\text { Edmonton }\end{array}$ & $\begin{array}{l}\text { Los pacientes en hemodiálisis presentan } \\
\text { una alta prevalencia de fragilidad } \\
(29,6 \%) \text {. La fragilidad está asociada } \\
\text { con malos resultados a corto plazo y } \\
\text { mayores tasas de mortalidad ( } 58,5 \% \\
\text { de pacientes frágiles murieron), } \\
\text { visitas a los servicios de emergencia } \\
\text { hospitalarios y hospitalización. }\end{array}$ & $\begin{array}{l}\text { STROBE: } \\
20 / 22 \\
\text { SJR: Q2 }\end{array}$ \\
\hline $\begin{array}{l}\text { Hornik and } \\
\text { Dulawa }^{36} ; \\
2019 ; \\
\text { Polonia. }\end{array}$ & $\begin{array}{l}\text { Estudio longitudinal } \\
\text { de cohorte prospectivo }\end{array}$ & 72 & HD & $\begin{array}{l}\text { CSHA-EFC. } \\
\text { Escala } \\
\text { fragilidad } \\
\text { clínica. }\end{array}$ & $\begin{array}{l}\text { La mayoría de los pacientes } \\
\text { diagnosticados de fragilidad no } \\
\text { siguieron la actividad física ( } 79,3 \% \text { ). } \\
\text { La calidad de vida fue mejor en } \\
\text { pacientes activos en comparación con } \\
\text { pacientes inactivos, especialmente en } \\
\text { los dominios del sueño y el rendimiento } \\
\text { físico. }\end{array}$ & $\begin{array}{l}\text { STROBE: } \\
20 / 22 \\
\text { SJR: Q2 }\end{array}$ \\
\hline $\begin{array}{l}\text { Chan et al. }{ }^{37} \text {; } \\
\text { 2020; China }\end{array}$ & $\begin{array}{l}\text { Estudio longitudinal } \\
\text { de cohorte prospectivo }\end{array}$ & 267 & DP & $\begin{array}{l}\text { Cuestionario } \\
\text { chino validado }\end{array}$ & $\begin{array}{l}44,9 \% \text { de los pacientes eran frágiles. } \\
\text { Los individuos frágiles eran más } \\
\text { propensos a tener obesidad central } \\
(p<0,001) \text {. Fragilidad y obesidad } \\
\text { central tiene un efecto aditivo sobre la } \\
\text { mortalidad. }\end{array}$ & $\begin{array}{l}\text { STROBE: } \\
21 / 22 \\
\text { SJR: Q1 }\end{array}$ \\
\hline $\begin{array}{l}\text { Jegatheswa- } \\
\text { ran et al.38; } \\
\text { 2020; } \\
\text { Canadá. }\end{array}$ & $\begin{array}{l}\text { Estudio longitudinal } \\
\text { de cohorte prospectivo }\end{array}$ & 261 & $\begin{array}{l}-D P(101) \\
-H D(135) \\
-H D \text { domiciliaria } \\
(25)\end{array}$ & Escala FRAIL & $\begin{array}{l}15 \% \text { eran frágiles y } 55 \% \text { prefrágiles. } \\
\text { Los pacientes frágiles tenían más } \\
\text { probabilidades de ser hospitalizados } \\
(p=0,01) \text { y pasar más tiempo en el } \\
\text { hospital }(p=0,04) \text {. }\end{array}$ & $\begin{array}{l}\text { STROBE: } \\
21 / 22 \\
\text { SJR: Q2 }\end{array}$ \\
\hline $\begin{array}{l}\text { Ali H. Ab- } \\
\text { delaziz T. et } \\
\text { al. } 39 ; 2018 ; \\
\text { Inglaterra. }\end{array}$ & $\begin{array}{l}\text { Estudio longitudinal } \\
\text { cruzado de cohorte } \\
\text { prospectivo }\end{array}$ & 104 & $\begin{array}{l}\text {-Prediálisis } \\
\text {-DP } \\
\text {-HD }\end{array}$ & $\begin{array}{l}\text { Cuestionario } \\
\text { PRISMA } \\
\text { y prueba } \\
\text { Levántate y } \\
\text { anda (TUGT) }\end{array}$ & $\begin{array}{l}\text { La supervivencia en pacientes frágiles } \\
\text { que iniciaron diálisis peritoneal (DP) } \\
\text { fue ligeramente mejor que los que } \\
\text { comenzaron hemodiálisis (HD) ( } p= \\
0,23 \text { ). La tasa de mortalidad fue } \\
\text { significativamente mayor en el grupo } \\
\text { frágil en comparación con el no frágil } \\
(p=0,01 \text { ). }\end{array}$ & $\begin{array}{l}\text { STROBE: } \\
20 / 22 \\
\text { SJR: Q3 }\end{array}$ \\
\hline $\begin{array}{l}\text { Pérez M. et } \\
\text { al. }{ }^{40} ; 2020 ; \\
\text { España. }\end{array}$ & $\begin{array}{l}\text { Estudio longitudinal } \\
\text { de cohorte prospectivo } \\
\text { multicéntrico }\end{array}$ & 2086 & $\begin{array}{l}\text { Pacientes en } \\
\text { diálisis en Lista } \\
\text { de trasplante }\end{array}$ & $\begin{array}{l}\text { Criterios de } \\
\text { Fried }\end{array}$ & $\begin{array}{l}\text { Añadiendo la interacción entre } \\
\text { comorbilidad y fragilidad a un modelo } \\
\text { de estimación del riesgo de mortalidad } \\
\text { mejoró significativamente la predicción, } \\
\text { aumentando el estadístico c de } 0,640 \text { a } \\
0,656(p<0,001) \text {. }\end{array}$ & $\begin{array}{l}\text { STROBE: } \\
20 / 22 \\
\text { SJR: Q3 }\end{array}$ \\
\hline $\begin{array}{l}\text { Konel et al. }{ }^{41} ; \\
\text { 2018; EE.UU. }\end{array}$ & $\begin{array}{l}\text { Estudio longitudinal } \\
\text { de cohorte prospectivo } \\
\text { y multicéntrico }\end{array}$ & 773 & $\begin{array}{l}\text { Pacientes en } \\
\text { diálisis en Lista } \\
\text { de trasplante }\end{array}$ & $\begin{array}{l}\text { Criterios de } \\
\text { Fried }\end{array}$ & $\begin{array}{l}\text { La coexistencia de síntomas depresivos } \\
\text { y fragilidad se asoció con una estancia } \\
\text { hospitalaria más larga }(p<0,001) \text {. } \\
\text { El efecto sinérgico de la fragilidad y } \\
\text { los síntomas depresivos solo tienen } \\
\text { resultados impactantes a corto plazo. }\end{array}$ & $\begin{array}{l}\text { STROBE: } \\
21 / 22 \\
\text { SJR: Q2 }\end{array}$ \\
\hline
\end{tabular}




\begin{tabular}{|c|c|c|c|c|c|c|}
\hline $\begin{array}{l}\text { Auto; Año; } \\
\text { País }\end{array}$ & Tipo de estudio & Muestra & $\begin{array}{l}\text { Tipo de } \\
\text { Tratamiento }\end{array}$ & $\begin{array}{l}\text { Instrumento } \\
\text { de medida }\end{array}$ & Resultados & $\begin{array}{l}\text { Criterio de } \\
\text { calidad }\end{array}$ \\
\hline $\begin{array}{l}\text { Chu et al.42; } \\
\text { 2019; EE.UU. }\end{array}$ & $\begin{array}{l}\text { Estudio longitudinal } \\
\text { de cohorte prospectivo }\end{array}$ & 569 & $\begin{array}{l}\text { Pacientes en } \\
\text { diálisis en Lista } \\
\text { de trasplante }\end{array}$ & $\begin{array}{l}\text { Criterios de } \\
\text { Fried }\end{array}$ & $\begin{array}{l}\text { Entre la evaluación y el TR, el } 22,0 \% \\
\text { se volvió más frágil, mientras que el } \\
24,4 \% \text { se volvió menos frágil. Los } \\
\text { candidatos que se volvieron más } \\
\text { frágiles tenían un mayor riesgo de } \\
\text { mortalidad post-TR y tenían mayores } \\
\text { probabilidades de una duración de la } \\
\text { estancia. }\end{array}$ & $\begin{array}{l}\text { STROBE: } \\
21 / 22 \\
\text { SJR: Q2 }\end{array}$ \\
\hline $\begin{array}{l}\text { McAdams-De- } \\
\text { Marco et } \\
\text { al.43; 2018; } \\
\text { EE.UU. }\end{array}$ & $\begin{array}{l}\text { Estudio longitudinal } \\
\text { de cohorte prospectivo }\end{array}$ & 223 & $\begin{array}{l}\text { Pacientes en } \\
\text { diálisis en Lista } \\
\text { de trasplante }\end{array}$ & $\begin{array}{l}\text { Criterios de } \\
\text { Fried }\end{array}$ & $\begin{array}{l}\text { En los modelos ajustados, solo la } \\
\text { fragilidad se asoció con la CVRS } \\
\text { regular o deficiente en } \\
\text { seguimiento (0R: } 2,79 \text {, IC del } 95 \% \text { : } \\
1,32-5,90 \text { ) y empeoramiento de la } \\
\text { CVRS en el seguimiento (RR: } 2,91 \text {, IC } \\
\text { del } 95 \%: 1,08-7,80 \text { ). }\end{array}$ & $\begin{array}{l}\text { STROBE: } \\
\text { 20/22 } \\
\text { SJR: Q2 }\end{array}$ \\
\hline $\begin{array}{l}\text { McAdams-De- } \\
\text { Marco et } \\
\text { al. }{ }^{44} ; 2017 ; \\
\text { EE.UU. }\end{array}$ & $\begin{array}{l}\text { Estudio de cohorte } \\
\text { prospectivo }\end{array}$ & 663 & $\begin{array}{l}\text { Pacientes en } \\
\text { diálisis en Lista } \\
\text { de trasplante }\end{array}$ & $\begin{array}{l}\text { Criterios de } \\
\text { Fried }\end{array}$ & $\begin{array}{l}\text { La edad fue el único factor } \\
\text { convencional asociado con la fragilidad } \\
\text { entre los receptores de TR; Sin } \\
\text { embargo, los factores que rara vez } \\
\text { se miden como parte de la práctica } \\
\text { clínica, como CVRS, AIVD y síntomas } \\
\text { depresivos, eran significativamente } \\
\text { correlativos a fragilidad. } 2 \text { patrones } \\
\text { de los } 5 \text { componentes, agotamiento y } \\
\text { velocidad de la marcha se asociaron con } \\
\text { la mortalidad post-TR. }\end{array}$ & $\begin{array}{l}\text { STROBE: } \\
20 / 22 \\
\text { SJR: Q1 }\end{array}$ \\
\hline
\end{tabular}

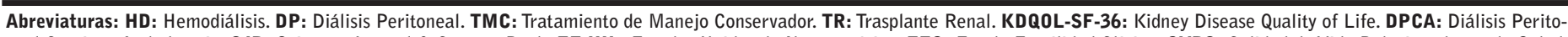

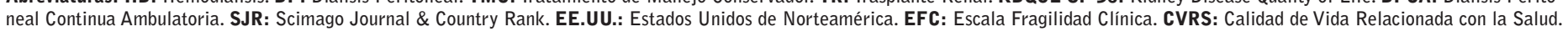
AIVD: Actividades Instrumentales para la Vida Diaria.

\section{Mortalidad y supervivencia}

La mortalidad es uno de los factores más estudiados y asociados a la fragilidad, en esta revisión se documenta en varios estudios que existe mayor tasa de mortalidad en los pacientes más frágiles, tanto en diálisis sin diferenciación de modalidad ${ }^{20,39}$ como en $\mathrm{HD}^{27,32,34}$. Kang et $a .^{30}$ en un estudio de cohorte prospectivo sobre 1250 pacientes en HD y 366 en DP, no encontraron diferencias de prevalencia de fragilidad entre ambos tratamientos, si bien concluyeron que la discapacidad fue más común en los pacientes en DP $(29,8 \%)$ que en los pacientes en HD $(15,6 \%)$, además en ambos tratamientos se asoció fragilidad a una disminución de supervivencia y supervivencia libre de hospitalización.

En un estudio transversal, con pacientes en HD la fragilidad fue medida de dos formas diferentes, los pacientes que cumplieron con la definición basada en el autoinforme, pero no con la definición de rendimiento de fragilidad, no tenían un riesgo de mortalidad estadísticamente mayor que los que no eran frágiles por ninguna definición, pero aquellos que cumplían con ambas definiciones de fragilidad tenían un riesgo significativamente mayor ${ }^{27}$.

En otro estudio con pacientes en DP, la fragilidad y deterioro cognitivo coexistentes se asociaron a una disminución de la supervivencia ${ }^{22}$. También se estudió en pacientes en diálisis en lista de trasplante, síntomas depresivos y fragilidad, y estos tenían 2,62 veces mayor riesgo de mortalidad, en comparación a los que no eran frágiles y no tenían síntomas depresivos ${ }^{41}$.

En algunos artículos en los que los pacientes en diálisis estaban incluidos en lista de espera para trasplante renal, analizaron la fragilidad antes de la inclusión en esta lista y en el momento de recibir el trasplante renal, y se observó que los candidatos que se volvieron más frágiles desde la evaluación hasta el trasplante tenían un mayor riesgo de mortalidad postrasplante ${ }^{42,44}$. Entre los 5 componentes del fenotipo de fragilidad, el agotamiento y la velocidad de marcha más lenta fueron los que se asociaron con más riesgo de mor- 
talidad, dos veces más entre todos los receptores de trasplante renal ${ }^{44}$. En otro estudio se midió fragilidad y comorbilidad y la relación de ambas con mortalidad, encontrando que los candidatos no frágiles con una alta carga de comorbilidad tenían un mayor riesgo de mortalidad en lista de espera, mientras que para los pacientes que ya eran frágiles la comorbilidad no era tan influyente respecto a mortalidad ${ }^{40}$.

\section{Tasa de hospitalización}

Varios estudios han documentado una asociación significativa de la fragilidad con la tasa de hospitalización, siendo la presencia de fragilidad indicativa de una mayor hospitalización en pacientes en diálisis ${ }^{20,38}$, tanto en $\mathrm{HD}^{25,31}$, como en DP29. Por otro lado, algunos estudios han documentado la relación de la duración de la estancia hospitalaria con la fragilidad, asociada a un mayor tiempo en diálisis ${ }^{38,29}$. En este estudio, en los pacientes en DP, la media de estancia hospitalaria fue de 8,04 días para pacientes no frágiles, mientras que, para los frágiles, fue de $14,05^{29}$.

Un estudio que comparaba la fragilidad entre pacientes mayores que entraban en tratamiento con diálisis frente a otro grupo que permaneció en tratamiento conservador o paliativo, encontró un $78 \%$ y $88 \%$ de fragilidad respectivamente, aunque las diferencias no fueron significativas. Sin embargo, la hospitalización ocurrió en el $50 \%$ de pacientes dializados y en el $24 \%$ de los pacientes en tratamiento conservador $(p<0,01)^{21}$. También un estudio relacionó una mayor tasa de hospitalización con la gravedad de la fragilidad en pacientes con HD $(p<0,01)^{25}$. Por otro lado, otro estudio concluyó que la hospitalización se asociaba independientemente con el empeoramiento de la fragilidad en pacientes en $H D^{31}$.

Además, en pacientes dializados en lista de trasplante, se evalúo el estado de fragilidad antes y en el momento del trasplante, el $22,0 \%$ de los pacientes se volvieron más frágiles. Los candidatos que se volvieron más frágiles tenían mayores probabilidades de una duración de la estancia hospitalaria $\geq 2$ semanas después de ser trasplantados ${ }^{42}$. Otro estudio, destacó que la coexistencia de depresión y fragilidad en los pacientes tuvo efecto sinérgico sobre la estancia hospitalaria, prolongándola $(p<0,001)^{41}$.

\section{Discapacidad}

En un análisis multivariado en pacientes en diálisis se encontraron asociaciones significativas de fragilidad con diversos factores, entre ellos la discapacidad que se observó en un $19,1 \%$ de los pacientes ${ }^{20}$. En otro estudio se mostró que para pacientes en HD y DP no había diferencias significativas respecto a ser frágiles, pero sí tenían más posibilidades de presentar discapacidad para actividades de la vida diaria aquellos que estaban en DP $(p<0,001)^{30}$.

Respecto a los pacientes en Lista de espera para trasplante, en un estudio la fragilidad se asoció de manera independiente a algunos factores, entre ellos la discapacidad para las actividades instrumentales de la vida diaria ${ }^{44}$.

\section{Deterioro cognitivo y síntomas depresivos}

La fragilidad también ha sido asociada con una peor función cognitiva, particularmente con la función cognitiva global, en pacientes en $H^{34}$. En esta misma línea, otro estudio en pacientes en DP demostró que el efecto sinérgico de fragilidad y deterioro cognitivo se relacionaba con menor supervivencia ${ }^{22}$.

Resultados similares se encontraron en un estudio en pacientes en DP sobre fragilidad y síntomas depresivos, en el que el $62,4 \%$ de los pacientes eran frágiles, y de estos el $43,2 \%$ tenían síntomas depresivos. A los 24 meses de seguimiento, la supervivencia fue del $86,6 \%$ para pacientes sin fragilidad, $71,4 \%$ para los frágiles, pero sin síntomas depresivos y $62,5 \%$ para frágiles con síntomas depresivos $(p=0,001)$. Además, la mediana de estancia hospitalaria de días por año de seguimiento fue de 8,04 para pacientes sin fragilidad, 14,05 para los frágiles, pero sin síntomas depresivos y 26,62 frágiles con síntomas depresivos $(p<0,0001)^{29}$.

Respecto a los pacientes en diálisis en lista de trasplante con síntomas depresivos, tenían más probabilidades de ser frágiles que los que no tenían síntomas depresivos $(p<0,001)$. Además, aquellos con síntomas depresivos y fragilidad tuvieron 1,88 veces mayor tiempo de estancia hospitalaria $(p<0,001)$ en comparación a los que no eran frágiles y no tenían síntomas depresivos ${ }^{41}$. En otro estudio la prevalencia de fragilidad fue mayor en los pacientes en lista de trasplante con depresión $(53,2 \% \text { frente a } 16 \% ; p<0,001)^{44}$.

\section{Obesidad y comorbilidad}

En varios estudios se ha demostrado asociaciones significativas de fragilidad con diversos factores, entre ellos comorbilidad y mayor índice de masa corporal en diálisis ${ }^{20,35}$. En una cohorte de pacientes en DP los pacientes frágiles tenían mayor puntuación en el Índice de Comorbilidad de Charlson ${ }^{29}$. 
Con respecto a la obesidad, en un estudio se muestra que los individuos frágiles en DP eran más propensos a tener obesidad central (perímetro abdominal mayor), pero no obesidad general. Este estudio también muestra el efecto aditivo sobre la mortalidad por fragilidad física y obesidad central en pacientes en DP ${ }^{37}$.

\section{Caídas o fracturas}

Con respecto a las caídas, diferentes autores coinciden en que los pacientes en HD frágiles tienen mayor probabilidad de caídas $^{25,28}$. Además, en uno de estos estudios la incidencia de eventos que requerían atención médica o fracturas fue el doble para pacientes frágiles que para los no frágiles ${ }^{28}$.

\section{Estudios y renta per cápita}

Un análisis multivariado demostró asociaciones significativas de fragilidad con varios factores, entre ellos un menor nivel educativo en pacientes en diálisis ${ }^{20}$. Otro estudio en pacientes en diálisis en lista de trasplante, asoció tener estudios menores a educación secundaria, como factor independiente a fragilidad (OR=3,10, IC del 95\%: $1,30-7,36)^{44}$.

Otro estudio transversal en pacientes en HD, analizó diversos factores asociados a la fragilidad (género, edad, color, renta mensual per cápita, duración de la diálisis, número de enfermedades asociadas, caídas año, nivel de hematocrito, hormona paratiroidea y uso de calcitriol), y encontraron que solo el ingreso per cápita mensual se asoció significativamente con fragilidad $(0 R=0,44$; IC $95 \% 0,1-0,9 ; p=0,04)$, mostrando que los ancianos con mayor riesgo eran los que tenían menores ingresos ${ }^{33}$.

\section{Actividad y rendimiento físico}

Se ha asociado la actividad física de los pacientes en diálisis con resultados favorables en salud, que incluyeron fragilidad, calidad de vida, discapacidad y agotamiento ${ }^{19}$. En otro estudio en pacientes con HD se relacionó ser frágil a una disminución de actividad física y de la velocidad de la marcha32.

Por otro lado, un estudio evaluó la adherencia a la actividad física recomendada a los pacientes en HD, obteniendo como resultado que el $79,3 \%$ de los pacientes frágiles no siguieron la actividad física, además los pacientes activos tenían una tasa significativamente menor de comorbilidad y una mejor calidad de vida que los pacientes inactivos ${ }^{36}$.

El patrón más común que tenían los pacientes de diálisis en lista de trasplante y frágiles era poca fuerza de agarre, baja actividad física y velocidad de la marcha más lenta ${ }^{44}$.

\section{Otros factores asociados de interés}

Se ha asociado también a fragilidad en pacientes en diálisis, otros factores menos relevantes, como peores habilidades motoras orales y función de deglución ${ }^{39}$, una concentración baja de testosterona en hombres en $\mathrm{HD}^{24}$, mayor pérdida ósea ${ }^{26}$, marcadores de inflamación ${ }^{31}$, mala calidad de vida ${ }^{44}$ y raza negra como menos frágil ${ }^{42}$.

\section{Instrumentos de medida de fragilidad empleados}

Para la evaluación de fragilidad se han utilizado diferentes instrumentos de medida, aunque el más utilizado con diferencia, ha sido el de los Criterios de Fried, tal como puede observarse en la tabla 2.

Tabla 2. Instrumentos de medida de fragilidad.

\begin{tabular}{|c|c|c|}
\hline $\begin{array}{l}\text { Instrumento de } \\
\text { medida }\end{array}$ & Administración & Referencias \\
\hline Criterios de Fried & $\begin{array}{l}\text { Administrado por } \\
\text { un profesional de } \\
\text { la salud }\end{array}$ & $\begin{array}{l}\text { Hemodiálisis: } 23,24, \\
25,26,27,29,32,34 \\
\text { Lista de trasplante: } 40 \text {, } \\
41,42,43,44\end{array}$ \\
\hline $\begin{array}{l}\text { Escala Fragilidad } \\
\text { Clínica (EFC) }\end{array}$ & $\begin{array}{l}\text { Administrado por } \\
\text { un profesional de } \\
\text { la salud }\end{array}$ & $\begin{array}{l}\text { Hemodiálisis: } 36 \\
\text { Diálisis peritoneal: } 22\end{array}$ \\
\hline $\begin{array}{l}\text { Escala fragilidad } \\
\text { de Edmonton } \\
\text { (EFS) }\end{array}$ & $\begin{array}{l}\text { Administrado por } \\
\text { un profesional de } \\
\text { la salud }\end{array}$ & Hemodiálisis: 33, 35 \\
\hline $\begin{array}{l}\text { Cuestionario } \\
\text { chino de fragilidad } \\
\text { validado }\end{array}$ & $\begin{array}{l}\text { Administrado por } \\
\text { un profesional de } \\
\text { la salud }\end{array}$ & $\begin{array}{l}\text { Diálisis peritoneal: } \\
29,37\end{array}$ \\
\hline $\begin{array}{l}\text { Cuestionario } \\
\text { autoinformado de } \\
\text { fragilidad }\end{array}$ & Autoadministrado & Hemodiálisis: 28 \\
\hline $\begin{array}{l}\text { Valoración } \\
\text { geriátrica }\end{array}$ & $\begin{array}{l}\text { Administrado por } \\
\text { un profesional de } \\
\text { la salud }\end{array}$ & Diálisis: 21 \\
\hline Escala FRAIL & $\begin{array}{l}\text { Administrado por } \\
\text { un profesional de } \\
\text { la salud }\end{array}$ & Diálisis: 38 \\
\hline $\begin{array}{l}\text { Cuestionario } \\
\text { PRISMA y prueba } \\
\text { Levántate y anda } \\
\text { (TUGT) }\end{array}$ & $\begin{array}{l}\text { Administrado por } \\
\text { un profesional de } \\
\text { la salud }\end{array}$ & Diálisis: 39 \\
\hline
\end{tabular}


La mayoría de los instrumentos de medida utilizados son administrados por el profesional de la salud, exceptuando un cuestionario de fragilidad autoinformado (no especificado). En este estudio sobre la asociación de fragilidad autoinformada con caídas y fracturas la prevalencia fue de $77 \%{ }^{28}$.

Solo en un estudio trasversal se analizó la fragilidad percibida y medida entre adultos sometidos a HD. Entre los pacientes frágiles solo el $42 \%$ fueron percibidos correctamente por su nefrólogo y $39,2 \%$ por su enfermera. Solo $4,9 \%$ se percibían frágiles a sí mismos, por lo que la fragilidad percibida no es un indicador adecuado de medida de fragilidad ${ }^{23}$.

\section{Discusión}

Con esta revisión se pretendía conocer la bibliografía existente sobre los factores asociados a la fragilidad en los pacientes en TRS con diálisis.

En la mayoría de los artículos utilizados en esta revisión, existe una elevada prevalencia de fragilidad en pacientes sometidos a diálisis, sin variación significativa según el tipo de tratamiento, siendo de un $45,4 \%$ en artículos de diálisis (no se especifica modalidad), un $38,04 \%$ en HD y $42,15 \%$ en DP. Estos resultados, coinciden con gran parte de la bibliografía existente al respecto, donde la prevalencia de fragilidad es el doble en pacientes con ERC en comparación con la población de referencia sin ERC ${ }^{45-47}$.

Los pacientes que se encuentran en lista de espera para trasplantarse de riñón, independientemente del procedimiento dialítico en el que se encuentran, presentan menor incidencia de fragilidad $(22,78 \%)$, algo lógico puesto que el ser menos frágil o no serlo, puede ser uno de los criterios de selección clínica para la inclusión en lista de trasplante renal. Como informa la última Memoria de actividad de donación y trasplante renal de 2019 , menos de un $25 \%$ de los pacientes en HD acceden a la lista de espera de trasplante en nuestro país ${ }^{48}$.

Como era de esperar, otro resultado significativo ha sido la estrecha relación de fragilidad con mortalidad, presente en una gran parte de los estudios en las diferentes modalidades de TRS; además, se ha visto el efecto sinérgico con otros factores, como el deterioro cognitivo y los síntomas depresivos, que unidos a la fragilidad, aumentan el riesgo de mortalidad en pacientes en DP22,29 y en lista de trasplante ${ }^{41}$. Existe numerosa bibliografía que corrobora esta asociación de fragilidad y mortalidad como ya demostró Johansen KL et al. ${ }^{3}$ en 2007 y otros autores en sus estudios originales en 2012,2013 y $2014^{49-51}$. Sin embargo, en uno de los estudios encontrados en pacientes en HD la gravedad de la fragilidad no se asocia a la mortalidad ${ }^{25}$ y en otro, ser frágil y tener comorbilidades no se asocia a mayor mortalidad en pacientes en lista de trasplante ${ }^{40}$; esto se puede interpretar como que existe un límite en el que no aumenta el riesgo de mortalidad por tener más gravedad de fragilidad ni por tener más comorbilidad, sino que ya existe ese riesgo por el hecho de ser frágil.

En cuanto a la tasa de hospitalización, en diferentes artículos de esta revisión se ha encontrado una relación significativa con la presencia de fragilidad, como mayor hospitalización, mayor duración de estancia hospitalaria y mayor gravedad de motivo de ingreso ${ }^{25}$, resultados coincidentes con las conclusiones de una revisión sistemática del año $2017^{12}$. Los pacientes en lista de trasplante que tenían mayor fragilidad antes del trasplante tenían un mayor riesgo de estancia hospitalaria ${ }^{41,42}$, en la línea de lo publicado por otros autores que comunicaron que los pacientes frágiles que se trasplantan, suelen tener una hospitalización más prolongada y con mayores complicaciones ${ }^{52-54}$.

Diferentes autores han relacionado la fragilidad de los pacientes en diálisis ${ }^{20,30}$ y en diálisis en lista de trasplante ${ }^{44}$ con discapacidad. Por otro lado, también se ha asociado fragilidad a síntomas depresivos tanto en pacientes en diálisis ${ }^{34,29}$ como en lista de trasplante ${ }^{41,44}$. Estos resultados coinciden con otros estudios de años anteriores que relacionaban fragilidad con discapacidad ${ }^{55}$, pérdida de función cognitiva y deterioro cognitivo ${ }^{57}$. Otros factores asociados a fragilidad destacables han sido la obesidad y comorbilidad $20,29,35$, relacionándose esta última con mayor mortalidad 37,40 , algo lógico, pues tanto la obesidad como mayor comorbilidad son factores de riesgo de mortalidad.

Todos estos factores asociados, discapacidad, deterioro cognitivo, síntomas depresivos, comorbilidad y obesidad podrían considerarse "elementos contribuyentes" a la aparición de fragilidad, como también la fragilidad podría precipitar uno de estos factores adversos ${ }^{6}$. Por lo tanto, sería importante una valoración integral de todos estos factores, para evitar así un deterioro más rápido en estos pacientes. De forma menos relevante se han asociado otros factores a fragilidad, como un 
mayor número de caídas ${ }^{25,28}$, menor nivel de estudios de los pacientes ${ }^{20,44}$ y renta per cápita ${ }^{33}$.

La actividad y rendimiento físico ha sido el único factor asociado a fragilidad en esta revisión que podemos decir que es beneficioso, disminuyendo la fragilidad en diálisis ${ }^{19}$. Así, los pacientes que participaban en la actividad física y eran frágiles en HD tenían una tasa significativamente menor de comorbilidad y una mejor calidad de vida que los pacientes inactivos ${ }^{36}$. De hecho, algunos estudios ponen de relieve como signos de fragilidad la disminución de actividad física y velocidad de la marcha ${ }^{32,44}$ y poca fuerza de agarre ${ }^{44}$. Por lo tanto, parece que los componentes más prevalentes encontrados en pacientes frágiles se podrían mejorar con cierta actividad física.

Por último, destacar que el instrumento más utilizado para medir la fragilidad en los pacientes en diálisis, son los Criterios de Fried, con gran diferencia. Los resultados pueden variar según el modo de administrar el instrumento de medida, como se aprecia en el estudio de Salter et al. ${ }^{23}$, ya que, dependiendo de la percepción del nefrólogo, enfermera y el propio paciente, la fragilidad percibida fue un indicador inadecuado de la fragilidad medida. Por ello, sería importante la instauración de un instrumento de medida de fragilidad más universal, 0 al menos que la comunidad científica recomendara la utilización del mismo instrumento a nivel internacional, para poder tener así una medición más objetiva y comparable.

\section{Limitaciones del estudio}

Las principales limitaciones de este estudio han sido la variabilidad en cuanto al tipo de TRS recibido por los pacientes, ya que esto ha dificultado el modo de estructurar los resultados y que el número de estudios encontrados en los que los pacientes estaban en HD ha sido mayor que los pacientes en DP y en Lista de trasplante.

\section{Consideraciones prácticas}

De la bibliografía encontrada en los últimos 5 años se desprende que la fragilidad es un síndrome multidimensional prevalente en las personas con ERC en diálisis, que se asocia con múltiples factores de riesgo. En este sentido, la enfermera puede tener un papel fundamental para prevenir estos factores de riesgo y en detectar precozmente la fragilidad, la cual no lleva mucho tiempo ni tiene gran dificultad técnica, y en segundo lugar, fomentando y ayudando a la realización de una mayor actividad física. Como instrumento de medida recomendaríamos los Criterios de Fried, puesto que es el más conocido y es administrado por un profesional de manera más objetiva.

A la vista de los resultados obtenidos en esta revisión podemos enumerar como principales conclusiones:

- La fragilidad es muy frecuente en el enfermo renal crónico, independientemente del TRS en el que se encuentre, aunque es menos prevalente en aquellos pacientes que están en lista de espera para trasplante renal.

- La fragilidad está asociada a diferentes factores de riesgo en los pacientes en TRS con diálisis, como mortalidad, más hospitalización, aumento de la duración de la estancia hospitalaria, discapacidad, deterioro cognitivo, síntomas depresivos, comorbilidad, obesidad y caídas.

- Otros resultados asociados a fragilidad en estos pacientes son la pérdida de masa ósea y marcadores de inflamación aumentados, así como menos renta per cápita y menor nivel de estudios. En el hombre puede estar asociado a bajos niveles de testosterona.

- Como factor protector de fragilidad, la actividad y rendimiento físico puede prevenir el estado de fragilidad e incluso mejorarlo, además de influir positivamente en la calidad de vida del paciente.

- El instrumento de medida de fragilidad más utilizado son los Criterios de Fried, administrado por profesionales sanitarios. Sería recomendable nuevos estudios de investigación y desarrollo de instrumentos de medida de fragilidad más unificados y universales.

Recepción: 02-08-21

Aceptación: 30-08-21

Publicación: 30-09-21

\section{Bibliografía}

1. Fried LP, Ferrucci L, Darer J, Williamson JD, Anderson $G$. Untangling the concepts of disability, frailty, and comorbidity: implications for improved targeting and care. J Gerontol Ser A. 2004; 59(3):M255-63. 
2. Portilla Franco ME, Tornero Molina F, Gil Gregorio P. La fragilidad en el anciano con enfermedad renal crónica. Nefrología (Madr.) [Internet]. 2016 [consultado 25 Ene 2021]; 36(6):609-15. Disponible en: http://scielo.isciii.es/scielo.php?script=sci_arttext\&pid=S0211-69952016000700609\&lng=es.

3. Johansen KL, Chertow GM, Jin C, Kutner NC. Significance of frailty among dialysis patients. JASN [Internet]. 2007 [consultado 25 Ene 2021]; 18 (11) 2960-7; Disponible en: https://doi.org/10.1681/ ASN.2007020221.

4. Martín I, Gorroñogoitia A, Gómez J, Baztán JJ, Abizanda P. El anciano frágil. Detección y tratamiento en AP. [Internet]. 2010 [consultado 25 Ene 2021];42(7)388-93; D0I: https://doi.org/ 10.1016/j.aprim.2009.09.022.

5. Dent $E$, Kowal $P, 0$. Hoogendijk E. Frailty measurement in research and clinical practice a review. [Internet]. 2016 [consultado 25 Ene 2021]; 31:310. Disponible en: https://www.ejinme.com/article/ S0953-6205(16)30027-9/fulltext.

6. Romero Cabrera AJ. Fragilidad y enfermedades crónicas en los adultos mayores. Med Int Mex; [Internet]. 2011 [consultado 29 Ene 2021]; 27(5):45562. Disponible en: https://www.medigraphic.com/ pdfs/medintmex/mim-2011/miml15g.pdf.

7. Bohm C, Storsley L, Tangri N. The assessment of frailty in older people with chronic kidney disease. Curr Opin Nephrol Hypertens. [Internet]. 2015 [consultado 29 Ene 2021]; 24. (6):498-504; Disponible en: https://journals.Iww.com/co-nephrolhypertens/Fulltext/2015/11000/The_assessment_ of_frailty_in_older_people_with.6.aspx.

8. Wilhelm-Leen ER, Hall YN, Tamura M, Chertow GM. Fragilidad y enfermedad renal crónica: Tercera Encuesta Nacional de Evaluación de la Salud y la Nutrición. Am J Med 2009;122:664-71.

9. McAdams-DeMarco MA, Suresh S, Law A, Salter $M L$, Giménez $L F$, et al. Fragilidad y caídas entre los pacientes adultos sometidos a hemodiálisis crónica: un estudio de cohorte prospectivo. BMC Nephrol. [Internet]. 2013 [consultado 30 Ene 2021]; 14:224; D0I: https://doi.org/10.1186/1471-236914-224.
10. AlfaadhelTA, Soroka SD, Kiberd BA, Landry D, Moorhouse $P$, Tennankore KK. Fragilidad y mortalidad en diálisis: evaluación de una escala de fragilidad clínica. Clin J Am Soc Nephrol. [Internet]. 2015 [consultado 30 Ene 2021]; 7;10(5):832-40; Disponible en: https://cjasn.asnjournals.org/content/10/5/832.

11. Fried LP, Tangen CM, Walston J, et al. Frailty in older adults: evidence for a phenotype. J Gerontol A Biol Sci Med Sci 2001, 56(3):M146-56.

12. Chowdhury R, Peel NM, Krosch M, Hubbard RE. Frailty and chronic kidney disease: A systematic review. Arch Gerontol Geriatr [Internet]. 2017 [consultado 5 Feb 2021]; 135-42. Disponible en: https:// doi.org/10.1016/j.archger.2016.10.007.

13. Weiss C0. Fragilidad y enfermedades crónicas en adultos mayores. [Internet] Clínicas de Medicina Geriátrica; 2011 [consultado 6 Feb 2021]; disponible en: https://www.geriatric.theclinics.com/article/ S0749-0690(10)00077-7/fulltext.

14. Walker SR, Gill K, Macdonald $\mathrm{K}$ et al. Asociación de fragilidad y función física en pacientes con ERC no diálisis: una revisión sistemática. BMC Nefrol. [Internet]. 2013 [consultado 8 Feb 2021];14:228. Disponible en: https://doi.org/10.1186/1471-2369-14-228.

15. Urrútia G, Bonfill X. Declaración PRISMA: una propuesta para mejorar la publicación de revisiones sistemáticas y metaanálisis. Med Clin. [Internet]. 2010 [consultado 29 Ene 2021]; 135(11):507-11. Disponible en: http://www.laalamedilla.org/Investigacion/Recursos/PRISMA\%20Spanish\%20Sept\%202010.pdf.

16. Moher D, LIberati A, Tetzlaff J, Altman DG. The Prisma Group. Ítems de referencia para publicar Revisiones Sistemáticas y Metaanálisis: La Declaración PRISMA. [Internet] fcsalud. 2016; [consultado 24 Feb 2021]; Disponible en: https://fcsalud. ua.es/es/portal-de-investigacion/documentos/herramientas-para-la-busqueda-bibliografica/declaracion-prisma.pdf.

17. Vandenbroucke JP, Von Elm E, Altman DG, Gotzsche PC, Mulrow CD, Pocock SJ, et al. Mejorar la comunicación de estudios observacionales en epidemiología (STROBE): explicación y elaboración. Gac Sanit. [Internet]. 2009; 23(2):1-28. Disponible en: https:// www.strobe-statement.org/fileadmin/Strobe/uploads/ translations/STROBE-Exp-SPANISH.pdf. 
18. Scimago Journal and Country Rank. Scimagojr [Internet]. 2020 [consultado 29 ene 2021]; Disponible en: https://www.scimagojr.com/.

19. Kang SH, Do JY, Jeong HY, Lee S, Kim JC. The Clinical Significance of Physical Activity in Maintenance Dialysis Patients. Kidney Blood Press Res [Internet]. 2017 [consultado 12 Feb 2021]; 42(3):575-86. Disponible en: http://dx.doi.org/10.1159/000480674.

20. Lee SY, Yang DH, Hwang E, Kang SH, Park SH, Kim TW, et al. The Prevalence, Association, and Clinical Outcomes of Frailty in Maintenance Dialysis $\mathrm{Pa}$ tients. J Ren Nutr [Internet]. 2017 [consultado 12 Feb 2021]; 27(2):106-12. Disponible en: https://doi. org/10.1053/j.jrn.2016.11.003.

21. Van Loon IN, Goto NA, Boereboom FTJ, Verhaar $M C$, Bots ML, Hamaker ME, et al.Quality of life after the initiation of dialysis or maximal conservative management in elderly patients: a longitudinal analysis of the Geriatric assessment in older patients starting Dialysis (GOLD) study. BMC Nephrology [Internet]. 2019 [consultado 13 Feb 2021]; 20. Disponible en: http://dx.doi.org/10.1186/s12882019-1268-3.

22. Yi C, Lin J, Cao P, Chen J, Zhou T, Yang R, et al. Prevalence and Prognosis of Coexisting Frailty and Cognitive Impairment in Patients on Continuous Ambulatory Peritoneal Dialysis. Scientific Reports (Nature Publisher Group) [Internet]. 2018 [consultado 16 Feb 2021];8:1-10. Disponible en: http://dx.doi. org/10.1038/s41598-018-35548-4.

23. Salter $M L$, Gupta $N$, Massie $A B$, McAdams-DeMarco MA, Law AH, Jacob RL, Giménez LF et al. Perceived frailty and measured frailty among adults undergoing hemodialysis: a cross-sectional analysis. BMC Geriatr [Internet]. 2015 [consultado 16 Feb 2021]; 15:52. Disponible en: https://doi. org/10.1186/s12877-015-0051-y.

24. Chiang JM, Kaysen GA, Segal M, Chertow GM, Delgado C, Johansen $\mathrm{K}$. Low testosterone is associated with frailty, muscle wasting and physical dysfunction among men receiving hemodialysis: a longitudinal analysis. Nephrol Dial Transplant [Internet]. 2019 [consultado 16 Feb 2021]; 34(5):802-10. Disponible en: https://www.ncbi. nlm.nih.gov/pmc/articles/PMC6503299/.
25. Yadla M, John JP, Mummadi M. A study of clinical assessment of frailty in patients on maintenance hemodialysis supported by cashless government scheme. Saudi journal of kidney diseases and transplantation [Internet]. 2017 [consultado 17 Feb 2021]; 28(1):15-22. Disponible en: http:// dx.doi.org/10.4103/1319-2442.198102.

26. Yoneki K, Kitagawa J, Hoshi K, Harada M, Watanabe $T$, Shimoda $T$, et al. Association between frailty and bone loss in patients undergoing maintenance hemodialysis. J Bone Miner Metab [Internet]. 2019 [consultado $17 \mathrm{Feb}$; 37(1):81-9; D0I: https://doi.org/ 10.1007/s00774-017-0898-4.

27. Johansen $K L$, Dalrymple LS, Glidden D, Delgado $C$, Kaysen GA, Grimes $B$, et al. Association of Performance-Based and Self-Reported Function-Based Definitions of Frailty with Mortality among Patients Receiving Hemodialysis. Clin J Am Soc Nephrol [Internet]. 2016 [consultado 18 Feb 2021]; 11(4):626-32. D0I: https://doi.org/ 10.2215/CJN.03710415.

28. Delgado C, Shieh $S$, Grimes B, Chertow GM, Dalrymple LS, Kaysen GA, et al. Association of Self-Reported Frailty with Falls and Fractures among $\mathrm{Pa}$ tients New to Dialysis. Am J Nephrol [Internet]. 2015 [consultado 19 Feb 2021]; 4 2(2):134-40. D0I: https://doi.org/10.1159/000439000.

29. Szeto CC, Chan GC, Ng JK, Chow KM, Kwan BC, Cheng PM, et al. Depression and Physical Frailty Have Additive Effect on the Nutritional Status and Clinical Outcome of Chinese Peritoneal Dialysis. Kidney Blood Press Res [Internet] 2018 [consultado 19 Feb 2021]; 06;43(3):914-23. Disponible en: http://dx.doi.org/10.1159/000490470.

30. Kang SH, Young Do J, Lee SY, Kim JC. Effect of dialysis modality on frailty phenotype, disability, and health-related quality of life in maintenance dialysis patients. PLoS One [Internet]; 2017 [consultado 22 Feb 2021] 05;12(5). Disponible en: http://dx.doi.org/10.1371/journal.pone.0176814.

31. Johansen KL, Dalrymple LS, Delgado C, Chertow GM, Selgas MR, Chiang J, et al. Factors Associated with Frailty and Its Trajectory among Patients on Hemodialysis. Clin J Am Soc Nephrol [Internet]. 2017 [consultado 23 Feb 2021]. D0I: https://doi. org/10.2215/CJN.12131116. 
32. Johansen $\mathrm{KL}$, Delgado $\mathrm{C}$, Kaysen GA, Chertow JC, Dalrymple LS, Segal MR, et al. Frailty Among Patients Receiving Hemodialysis: Evolution of Components and Associations With Mortality. J Gerontol A Biol Sci Med Sci [Internet]; 2019 [consultado 24 Feb 2021]; 74(3):380-6. DOI: https://doi.org/10.1093/gerona/gly206.

33. Gesualdo GD, Zazzetta MS, Say KG, Orlandi FdS. Factors associated with the frailty of elderly people with chronic kidney disease on hemodialysis. Ciência \& Saúde Coletiva [Internet]; 2016 [consultado 25 Feb 2021]; 21(11). Disponible en: http://dx.doi.org/10.1590/1413812320152111.18222015.

34. McAdams-DeMarco MA, Tan J, Salter ML, Gross A, Meoni LA, Jaar BG, et al. Frailty and Cognitive Function in Incident Hemodialysis Patients. Clin J Am Soc Nephrol [Internet]; 2015 [consultado 1 Mar 2021]; 10(12):2181-9. D0I: https://doi.org/10.2215/ CJN.01960215.

35. García-Cantón C, Ródenas A, López-Aperador C, Rivero $Y$, Antón $G$, Monzón $T$ et al. Frailty in hemodialysis and prediction of poor short-term outcome: mortality, hospitalization and visits to hospital emergency services. Ren Fail [Internet]; 2019 [consultado 2 de Mar 2021]; 41(1):567-75. DOI: https://doi.org/10.1080/0886022X.2019.1628061.

36. Hornik B, Duława J. Frailty, Quality of Life, Anxiety, and Other Factors Affecting Adherence to Physical Activity Recommendations by Hemodialysis Patients. Int J Environ Res Public Health [Internet]; 2019 [consultado 2 Mar 2021]; 16(10):1827. D0I: https:// doi.org/10.3390/ijerph16101827.

37. Chan GC, Jack Kit-Chung NG, Chow K, Kwong VW, Wing-Fai Pang, Cheng PM, et al. Interaction between central obesity and frailty on the clinical outcome of peritoneal dialysis patients. PLoS One [Internet]; 2020 [consultado 4 Mar 2021]; 15(10). Disponible en: http://dx.doi.org/10.1371/ journal.pone.0241242.

38. Jegatheswaran J, Chan R, Hiremath $S$, Moorman D, Suri RS, Ramsay T, et al. Use of the FRAIL Questionnaire in Patients With End-Stage Kidney Disease. Canadian Journal of Kidney Health \& Disease [Internet]; 2020 [consultado el 8 Mar 2021]; 7. Disponible en: http://dx.doi.org/10.1177/2054358120952904.
39. Ali H, Abdelaziz T, Abdelaal F, Baharani J. Assessment of prevalence and clinical outcome of frailty in an elderly predialysis cohort using simple tools. Saudi journal of kidney diseases and transplantation: an official publication of the Saudi Center for Organ Transplantation, Saudi Arabia [Internet]; 2018 [consultado 9 Mar 2021]; 29(1):63-70. Disponible en: http://dx.doi.org/10.4103/13192442.225175.

40. Pérez MF, Martínez Miguel $P$, Ying $H$, Haugen CE Chu NM, Rodríguez-Puyol DM, et al. Comorbidity, Frailty, and Waitlist Mortality among Kidney Transplant Candidates of All Ages. Am J Nephrol [Internet]; 2019 [consultado 16 Mar 2021]; 49(2):103 10. D0I: https://doi.org/ 10.1159/000496061.

41. Konel JM, Warsame F, Ying $\mathrm{H}$, Haugen CE, Mountford A, Chu NM, et al. Depressive symptoms, frailty, and adverse outcomes among kidney transplant recipients. Clin Transplant. 2018 [consultado 18 Mar 2021]; 32(10): el3391. D0I: https://doi.org/ $10.1111 /$ ctr.13391.

42. Chu NM, Deng $A$, Ying $H$, Haugen $C E$, Garonzik Wang JM, Segev DL, et al. Dynamic Frailty Before Kidney Transplantation: Time of Measurement Matters. Transplantation. 2019 [consultado 18 Mar 2021]; 103(8):1700-4. D0I: https://doi. org/10.1097/TP.0000000000002563.

43. McAdams-DeMarco MA, Ying $H$, Olorundare $I$, King EA, Desai $N$, Dagher $N$, et al. Frailty and Health-Related Quality of Life in End Stage Renal Disease Patients of All Ages. J Frailty Aging. 2016 [consultado 20 Mar 2021]; 5(3):174-9.

44. McAdams-DeMarco MA, Ying $\mathrm{H}$, Olorundare I, King EA, Haugen $C$, Buta $B$, Gross $A L$, et al. Individual Frailty Components and Mortality in Kidney Transplant Recipients. Transplantation. 2017 [consultado 27 Mar 2021]; 101(9):2126-32. D0I: https://doi. org/10.1097/TP.0000000000001546.

45. Roshanravan $B$, Khatri $M$, Robinson-Cohen $C$, Levin $\mathrm{G}$, Patel KV, de Boer IH, et al. A prospective study of frailty in nephrology-referred patients with CKD. Am J Kidney Dis [Internet]. 2012 [consultado 12 Abr 2021]; 60: 912-21. Disponible en: https://www.ajkd.org/article/S02726386(12)00854-2/fulltext. 
46. Dalrymple $L S$, Katz $R$, Rifkin $D E$, Siscovick $D$, Newman $A B$, Fried $L F$, et al. Kidney function and prevalent and incident frailty. Clin $\mathrm{J} \mathrm{Am} \mathrm{Soc}$ Nephrol [Internet]. 2013 [consultado $13 \mathrm{Abr}$ 2021]; 8(12):2091-9. Disponible en: https://pubmed.ncbi.nlm.nih.gov/24178972/.

47. Shlipak MG, Stehman-Breen $C$, Fried LF, Song $X$, Siscovick $D$, Fried $L P$, et al. The presence of frailty in elderly persons with chronic renal insufficiency. Am J Kidney Dis [Internet]. 2004 [consultado 19 Abr 2021]; 43: 861-7. Disponible en: https:// www.sciencedirect.com/science/article/abs/pii/ S0272638604001398.

48. Organización Nacional de Trasplante, Memoria de Donación y Trasplante Renal. [Internet] España 2019 [consultado $25 \mathrm{Abr} 2021$ ]. Disponible en: http://www.ont.es/infesp/Memorias/Actividad_de_ Donaci\%C3\%B3n_y_Trasplante_Renal_2019.pdf.

49. Rodríguez-Villareal I, Ortega 0 , Hinostroza J, Cobo G, Gallar P, Mon C, et al. Geriatric assessment for therapeutic decision-making regarding renal replacement in elderly patients with advanced chronic kidney disease Nephron Clin Pract [Internet]. 2014 [consultado 20 Abr 2021]; pp. 73-8. Disponible en: https://doi.org/10.1159/000363624.

50. McAdams-DeMarco, A. Law, M. Salter, B. Boyarski, L. Giménez L, Jaar BG, et al. Frailty as a Novel Predictor of Mortality and Hospitalization in $\mathrm{He}$ modialysis Patients of All Ages. J Am Geriatr Soc [Internet]. 2013 [consultado $20 \mathrm{Abr} 2121$ ]; pp. 896-901. Disponible en: https://doi.org/10.1111/ jgs.12266.

51. Roshanravan B, Khatri M, Robinson-Cohen C, Levin G, Patel K, De Boer IH, et al. A prospective study of frailty in nephrology-referred patients with CKD. Am J Kidney Dis. [Internet]; 2012 [consultado 20 Abr 2021] pp. 912-21. Disponible en: https://doi. org/10.1053/j.ajkd.2012.05.017.
52. Kovesdy CP, Furth $S$, Zoccali $C$, et al. Obesidad y enfermedad renal: consecuencias ocultas de la epidemia. Revista nefrología [Internet]; 2017 [consultado 26 Abr 2021]. Disponible en: https://www.revistanefrologia.com/es-obesidad-enfermedad-renal-consecuencias-ocultas-articulo-S0211699517300553.

53. Schopmeyer L, EI Moumni M, Nieuwenhuijs-Moeke GJ, Berger SP, Bakker SJL, Pol RA. Frailty has a significant influence on postoperative complications after kidney transplantation-a prospective study on short-term outcomes. Transpl Int [Internet]; 2019 [consultado $28 \mathrm{Abr} 2021]$; 32(1):66-74. DOI: https://doi.org/10.1111/tri.13330.

54. Dos Santos Mantovani $M$, Coelho de Carvalho $N$ Modelli de Andrade LG, Fereira-Filho SP, De Souza-Calvacante $\mathrm{R}$, et al. Frailty predicts surgical complications after kidney transplantation. A propensity score matched study. PLoS One [Internet]. 2020 [consultado 2 mayo 2021]; 15(2):e0229531. D0I: https://doi.org/10.1371/journal.pone.0229531.

55. Fried LP, Bandeen-Roche K, Kasper JD, Guralnik JM. Association of comorbidity with disability I older women: The Women's Health and Aging Study. $\mathrm{J}$ Clin Epidemiol [Internet]. 1999 [consultado 6 May 2021]; 28:153-9. Disponible en: https://doi. org/10.1016/s0895-4356(98)00124-3.

56. Hervás A, García de Jalón E. Situación cognitiva como condicionante de fragilidad en el anciano: Perspectiva desde un centro de salud. Anales Sis San Navarra [Internet]. 2005 [consultado 9 May 2021]; 28 (1):35-47. Disponible en: http:// scielo.isciii.es/scielo.php?script=sci_arttext\&pi$d=$ S1137-66272005000100004\&lng=es.

57. Enfermedad renal provoca deterioro cognitivo incluso en etapas tempranas. Infosalus [Internet]. 2017 [consultado 7 de May]. Disponible en: https:// www.infosalus.com/salud-investigacion/noticia-enfermedad-renal-provoca-deterioro-cognitivo-incluso-etapas-tempranas-20190411125114.html.

Este artículo se distribuye bajo una Licencia Creative Commons Atribución-NoComercial 4.0 Internacional. https://creativecommons.org/licenses/by-nc/4.0/ 